\title{
Análisis experimental de la difusión salina durante la desalación por congelación
}

\section{Experimental analysis of saline diffusion during freezing desalination}

\author{
CASTILLO-TÉLLEZ, Beatriz ${ }^{1} \uparrow$, CASTILLO-TÉLLEZ-Margarita ${ }^{2 *}$, LÓPEZ-VIDAÑA, Erick César ${ }^{3}$ y \\ MEJÍA-PÉREZ, Gerardo Alberto ${ }^{4}$
}

${ }^{1}$ Centro Universitario del Norte, Universidad de Guadalajara, Colotlán, Jalisco 46200 México

${ }^{2}$ Universidad Autónoma de Campeche, Facultad de Ingeniería, Campeche. C.P. 24085

${ }^{3}$ Universidad Nacional Autónoma de México, Instituto de Energías Renovables, Priv. Xochicalco S/N Temixco, Morelos México, C.P. 62580

${ }^{4}$ Universidad de Guadalajara, Coordinación General de Extensión, J. Guadalupe. Montenegro 2216, Col. Lafayette, Guadalajara, Jal. C.P. 44150

ID $1^{\text {er }}$ Autor: Beatriz, Castillo-Téllez / ORC ID: 0000-0003-3747-6320, Researcher ID Thomson: S-2264-2018, CVU CONACYT ID: 210564

ID $1^{\text {er }}$ Coautor: Margarita, Castillo-Téllez / ORC ID: 0000-0001-9639-1736, Researcher ID Thomson: S-2283-2018, CVU CONACYT ID: 210428

\author{
ID $2^{\text {do }}$ Coautor: Erick César, López-Vidaña / ORC ID: 0000-0002-9200-6578, CVU CONACYT ID: 333477 \\ ID $3^{\text {er }}$ Coautor: Mejía Pérez, Gerardo-Alberto
}

DOI: $10.35429 / J O E S .2019 .18 .6 .1 .7$

Recibido 9 de Enero, 2019; Aceptado 30 de Marzo, 2019

\begin{abstract}
Resumen
Las consecuencias sobre la salud y la baja calidad de vida causadas por el problema de la escasez de agua, así como la alta relación entre la energía y el abastecimiento de agua, llaman la atención hacia la desalación por congelación ( $\mathrm{F} / \mathrm{M}$, por sus siglas en inglés), una técnica bien conocida pero no utilizada para esta aplicación. Es considerada como la más sostenible en términos de demanda energética comparada con las técnicas de desalación comercial (aproximadamente 70\% menos que la desalación térmica). Sin embargo, presenta algunos problemas de "encapsulamiento" salino en el hielo, por lo que, en este trabajo, se lleva a cabo un análisis experimental para lograr una mejor comprensión del desplazamiento salino, utilizando una solución acuosa de cloruro de sodio, congelado a diferentes temperaturas mediante desalación $\mathrm{F} / \mathrm{M}$ progresiva. Se ha encontrado que, a una temperatura de congelación de $8^{\circ} \mathrm{C}$, es posible purificar hasta un promedio de $78,12 \%$ de la solución de sal congelada, obteniendo una salmuera fácil de separar.
\end{abstract}

Desalación por congelación, Agua salobre, Análisis experimental

\begin{abstract}
The consequences on health and low life quality caused by the problem of water scarcity, as well as the interrelation between energy and water supply, call attention to water desalination by Freezing Melting (F/M), which is a wellknown technique but unfortunately, not used for this application, which is considered the most sustainable, in terms of its lower energy demand compared with commercial desalination techniques (about $70 \%$ less than thermal desalination). However, it presents some problems regarding the saline trapping in the ice, therefore, in this work, an experimental analysis is carried out to achieve a better understanding of the saline displacement, using an aqueous solution of sodium chloride, frozen at different temperatures, and configurations containers, with a F/M progressive desalination. It has been found that, at a freezing temperature of $8^{\circ} \mathrm{C}$, it is possible to purify up to an average of $78.12 \%$ of the frozen salt solution, obtaining an easyto-separate brine.
\end{abstract}

Freezing desalination, BVrackish water, Experimental analysis

Citación: CASTILLO-TÉLLEZ, Beatriz, CASTILLO-TÉLLEZ-Margarita, LÓPEZ-VIDAÑA, Erick César y MEJÍAPÉREZ, Gerardo Alberto. Análisis experimental de la difusión salina durante la desalación por congelación. Revista de Sistemas Experimentales. 2019. 6-18: 1-7

$\uparrow$ Investigador contribuyendo como primer autor. 


\section{Introducción}

Después de muchos años de suministro a la población a través de la explotación del agua en acuíferos y aguas superficiales, estas fuentes se han vuelto cada vez más inaccesibles, costosas e insostenibles. Los acuíferos han sido sobreexplotados y los ríos y lagunas contaminados. Debido a esto, la capacidad de desalación instalada en el mundo ha aumentado rápidamente en la última década. Se ha logrado una reducción significativa del costo de la desalación como resultado de importantes avances tecnológicos (Ghaffour, Missimer, \& Amy, 2013).

Esto ha dado lugar a una disponibilidad a precios competitivos con las opciones de obtención de recursos hídricos convencionales (Ghaffour et al., 2013; Reddy \& Ghaffour, 2007). De hecho, recientemente se ha logrado una rápida instalación de plantas desaladoras a escala mundial, como adaptación a los efectos del cambio climático a los que contribuyen las tecnologías utilizadas para el suministro de agua dulce (L. Gao, Yoshikawa, Iseri, Fujimori, \& Kanae, 2017). Sin embargo, esta técnica requiere una alta cantidad de energía, debido a la alta salinidad del agua marina o salobre ( $\mathrm{Li}$, Goswami, \& Stefanakos, 2013).

El desarrollo de métodos de desalación energéticamente eficientes es importante para resolver estos problemas complejos. Sin embargo, las aplicaciones comerciales no se han desarrollado lo suficiente y los estudios son limitados a nivel laboratorio (Williams, Ahmad, Connolly, \& Oatley-Radcliffe, 2015).

Desde el punto de vista de la industria, el proceso congelación tiene una serie de ventajas importantes (Ahmad \& Williams, 2011; Ghaffour et al., 2013; Karagiannis \& Soldatos, 2008; M.S. Rahman, M. Ahmed, 2007; Peñate \& García-Rodríguez, 2012): Factor de separación muy alto, alta eficiencia energética (ya que el calor latente de congelación del agua es bajo comparado con el calor latente de la evaporación; $333.5 \mathrm{~kJ} / \mathrm{kg}$ y $2256.7 \mathrm{~kJ} / \mathrm{kg}$, respectivamente) (Rogers \& Mayhew, 1995), insensible a la suciedad biológica y la corrosión, la ausencia de pretratamiento químico, baja descarga de sustancias químicas tóxicas al medio ambiente y materiales de construcción baratos, que reducen el costo de capital (Williams et al., 2015).
Sin embargo, el problema más difícil de resolver es el atrapamiento de sal en el hielo durante el proceso de congelación (Cole \& Shapiro, 1998). Esto dificulta su separación y requiere el aplastamiento de hielo, por lo tanto, los costos de operación aumentan (Subramani \& Jacangelo, 2015; Wiegandt \& Berg, 1980).

La calidad de la separación de la sal del agua depende de varios factores relacionados principalmente con la cinética del proceso de congelación, incluso independientemente de la solubilidad de la sal en el hielo (Jungwirth, Vrbka, \& Jungwirth, 2005). En la literatura se encuentran muchos trabajos que se han centrado en obtener una menor salinidad del agua. Se ha propuesto usar agitadores y ventiladores para hacer circular la solución y el aire frío, mejorando la transferencia de masa y calor. Se ha integrado una "semilla" de hielo para lograr una congelación más rápida (Shirai, Wakisaka, Miyawaki, \& Sakashita, 1998). Yu et al. (Yu, Ma, \& Zhang, 2007) encontraron que a baja temperatura inicial de la solución (aproximadamente $6^{\circ} \mathrm{C}$ ), la concentración de la solución y el proceso de recristalización son factores importantes para obtener una pureza mayor del hielo producido.

También se han llevado a cabo experimentos a diferentes concentraciones de sal, agitando el recipiente de y también se ha aumentado el número de ciclos de lavado del hielo para determinar si los parámetros mencionados pueden reducir los efectos sobre la retención de sal y el uso de energía para congelar el agua (Rahman, Ahmed, \& Chen, 2007). Desafortunadamente, incluso con los progresos realizados en estas investigaciones, el fenómeno del rechazo de la sal/impureza en la interfaz sólido-líquido se mantuvo lejos de entender (Grange, Viskanta, \& Stevenson, 1976).

En este trabajo se llevaron a cabo tres tipos de experimentos para una mejor comprensión de la difusión salina, utilizando un aislamiento térmico en las paredes de los contenedores (Figura 1) para lograr un gradiente salino y térmico unidireccional, lo cual no se ha reportado en literatura revisada. Esta técnica conduce a la purificación de agua congelada con una fácil separación de la salmuera líquida. 
Además, se analizaron los perfiles tanto de temperatura como de salinidad, proporcionando información sobre la difusión de la sal, que se considera un factor importante para producir una calidad de agua dulce aceptable.

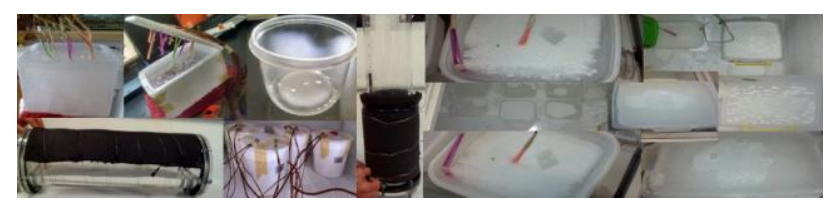

Figura 1 Tamaños, geometrías y aislamiento utilizado

\section{Metodología y experimentación}

En este artículo se realizaron tres experimentos:

- Se aislaron diferentes paredes de los contenedores utilizados para determinar cómo se vio afectada la difusión de iones salinos (ver Figura 1).

El $2^{\text {do }}$ experimento se basó en el modelo teórico unidimensional propuesto por (Castillo Téllez, 2017). La evolución de ambos procesos: las difusiones térmicas y de masa, se comparan de tal manera que este modelo puede predecir si la sal permanecerá dentro del hielo o la difusión de iones salinos tendrá lugar antes del cambio de fase. De acuerdo con esto, se ha realizado un experimento para representar y validar este modelo.

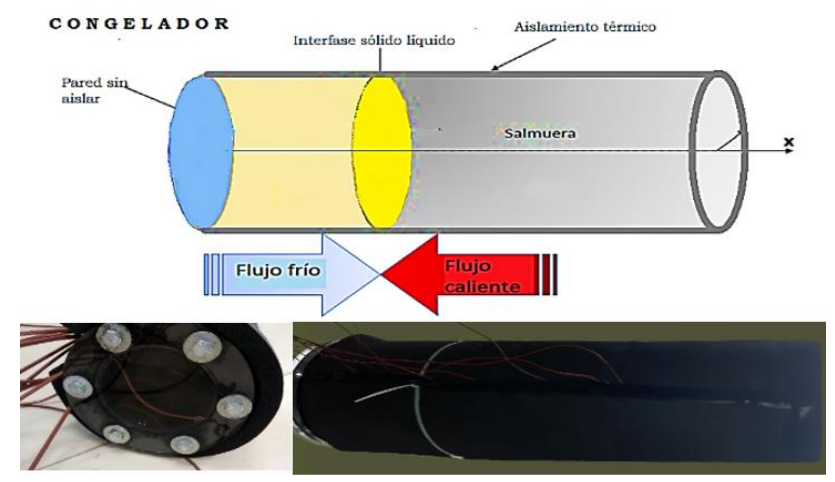

Figura 2 Modelo físico

Este proceso se denomina congelación progresiva. Es un método para concentrar las impurezas en una fase líquida y obtener una fase sólida pura mediante el control de un frente de hielo de forma unidimensional. Por lo tanto, permite la fácil separación del hielo y la salmuera concentrada. Sus principales aplicaciones son la industria alimentaria y farmacéutica (Chabarov \& Aider, 2014; W. Gao $\&$ Shao, 2009; Halde, 1980; Shirai et al., 1998).
Además, se han realizado estudios exitosos que demuestran la viabilidad de su uso para la desalación de agua (Fujioka, Wang, Dodbiba, \& Fujita, 2013).

Para simplificar la experimentación y obtener mediciones precisas, utilizamos cloruro de sodio $(\mathrm{NaCl})$ para estimar el comportamiento de la salmuera como la evolución del agua de mar. El cloruro de sodio es el componente principal del agua de mar y sus valores de densidad, viscosidad dinámica y calor específico son similares a los valores de agua de mar (A. Melinder \& Ignatowicz, 2015).

Este diseño físico consiste en un cilindro horizontal con aislamiento térmico excepto en un lado plano para impulsar el flujo de calor en una dirección X unidimensional (Figura 3). La superficie no aislada fue expuesta al aire frío a temperaturas por debajo del punto de congelación de la solución salina. El agua que progresivamente es congelada empieza a ser liberada de sal, enviándola a la salmuera líquida, cada vez más concentrada.

Dependiendo de las temperaturas de la salmuera y el aire frío, la evolución de la formación de hielo tiene lugar en la interfaz sólido-líquido. Cuando la temperatura del aire es inferior al punto de congelación de la solución salina y la temperatura de salmuera es mayor que la de la interfaz sólido-líquido, la transferencia de calor debería realizarse de la salmuera líquida hacia aire frío.

A través del hielo formado y del espesor del material del cilindro existe transferencia de calor por convección y por el aire frío y la salmuera, la transferencia es por convección natural.
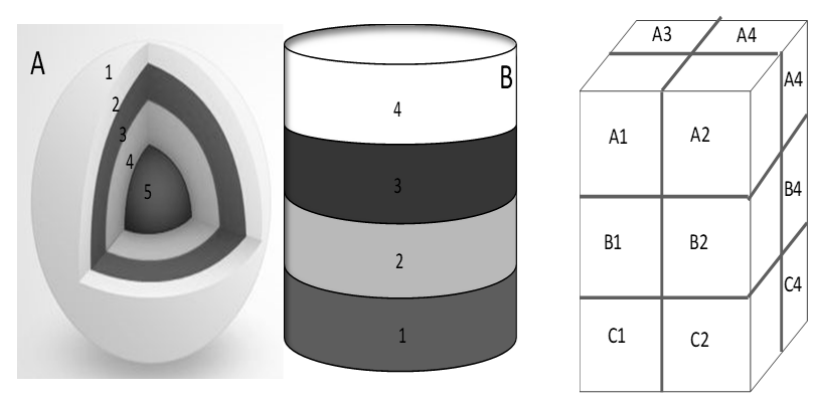

Figura 3 Sectores analizados para diferentes geometrías. a) tipo $\mathrm{A}, \mathrm{b}$ ) tipo $\mathrm{B}$ y c) tipo $\mathrm{C}$ 
- Por último, se probaron diferentes tamaños de contenedores y geometrías, para definir cómo afectan estos parámetros a la difusión salina. Para determinar la dirección de la difusión iónica, el hielo obtenido al final del proceso de desalinización de congelación se analizó en diferentes tipos de secciones (A, B and C), como se observa en la Figura 3.

Los tres experimentos tuvieron los siguientes pasos comunes:

- $\quad$ Preparación de la solución $\left(\mathrm{NaCl}-\mathrm{H}_{2} \mathrm{O}\right)$ con una concentración aproximada de $34.5 \mathrm{gr} / \mathrm{L}$.

Congelación a diferentes temperaturas. Se utilizó un congelador Scientemp Corp con una precisión de $\pm 1^{\circ} \mathrm{C}$.

- Monitorización del comportamiento térmico mediante 25 termopares colocados en todo el volumen del contenedor.

Para cortar el hielo formado y analizarlo, se diseñó un dispositivo con resistencias hechas de alambre de Kanthal, (aprovechando su resistencia al choque térmico manteniendo una alta resistencia eléctrica) como se muestra en la Figura 4.

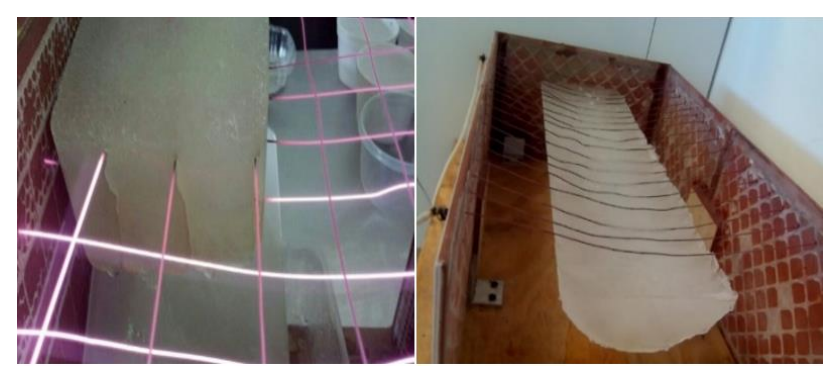

Figura 4 Proceso de corte de hielo

Dividimos nuestro hielo en segmentos que están numerados desde la pared sin aislamiento, hasta el último hielo formado, como se aprecia en la Figura 4.

Análisis de salinidad en diferentes secciones del hielo formado y en la salmuera líquida, utilizando un conductímetro marca Orion Star $^{\mathrm{TM}}$ A212 y un salinómetro marca Atago.

Los experimentos se realizaron a tres temperaturas diferentes: $-8,-15$ y $-30{ }^{\circ} \mathrm{C}$.

\section{Resultados y discusión}

La Figura 5 muestra la difusión salina en función del sector del contenedor utilizando diferentes longitudes del contenedor.

Para todos los valores de longitud (largo de los contenedores utilizados), la difusión salina aumenta con el aumento del segmento de contenedor, alcanzando su máximo en el último segmento. Además, hay una longitud crítica $\left(\mathrm{L}_{\mathrm{c}}=50 \mathrm{~cm}\right)$ después de la cual, la difusión salina no se puede mejorar. Este resultado coincide con lo encontrado por (Johnson, 1979).
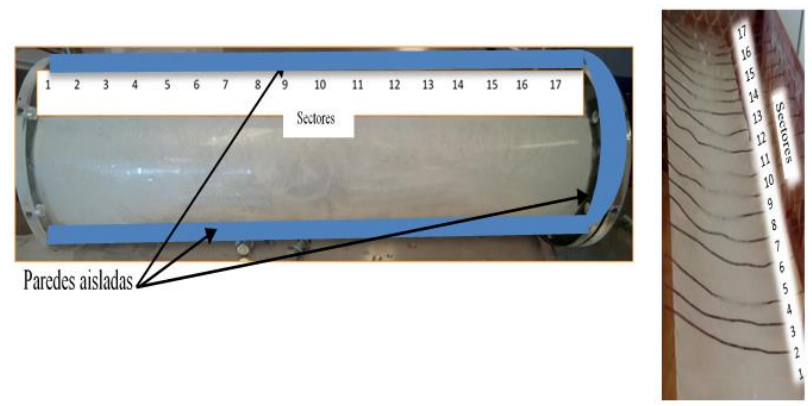

Figura 5 Procedimiento de corte y numeración de segmentos

El impacto de la velocidad de congelación se representa en figura 6. El mayor porcentaje de sal se desplaza en la misma dirección del cambio de fase sólido-líquido, de tal manera que los iones salinos se encuentran en el último segmento que se congeló o en la salmuera que no se logró congelar, dado su bajo punto de congelación.

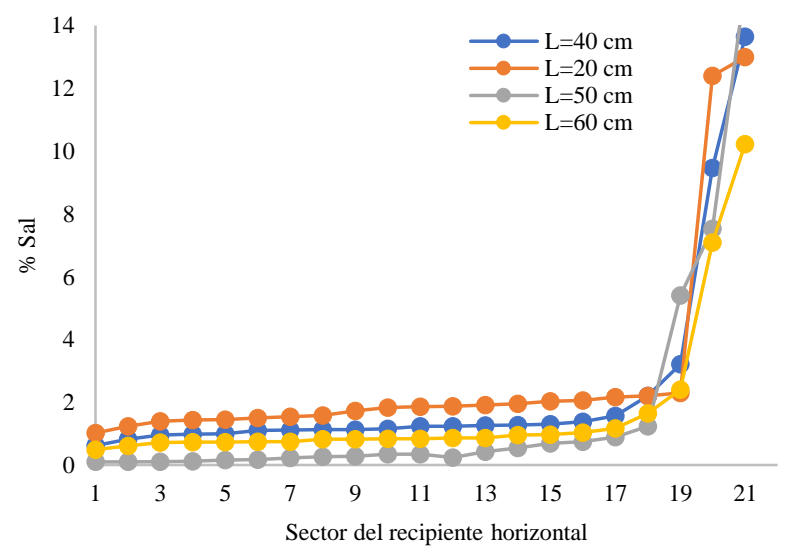

Figura 6 Salinidad para diversas longitudes, $T=-8^{\circ} \mathrm{C}$

Cuando la temperatura de congelación es muy baja, los iones salinos quedan atrapados dentro del hielo, porque la difusión salina es más lenta que la difusión térmica, por lo tanto, la desalación del agua no se puede lograr. 
Por lo tanto, la temperatura puede afectar a la difusión salina la cual se puede mejorar de una manera notable cuando la velocidad de congelación es menor $\left(-8^{\circ} \mathrm{C}\right)$. A esta temperatura, es posible desplazar $78.8 \%$ de sal del hielo hacia la salmuera concentrada. (140.13 gr de un volumen de $5.15 \mathrm{~L}$ de hielo formado a un $0.64 \mathrm{~L}$ de salmuera).

A $-30^{\circ} \mathrm{C}$, no se logra la desalación, por lo que, los iones salinos permanecen dentro del hielo formado, dado que el cambio de fase líquido-sólido se produce más rápido que la difusión de la sal. A velocidades de congelación más bajas, la difusión de la salina es mejor, y la sal es repelida hacia un sector específico que será más fácil de separar. Es importante mencionar que en el experimento a $-8^{\circ} \mathrm{C}$, cuando la concentración salina fue $17 \%$, la salmuera permanece líquida porque el punto de congelación de una solución diluida con concentraciones tan altas es muy bajo (el punto eutéctico para las soluciones de $\mathrm{NaCl}$ es a 23.4 $\%,-21^{\circ} \mathrm{C}$ ) (Ake Melinder, 2007) e incluso a $20^{\circ} \mathrm{C}$, es imposible congelar el agua salada en estas condiciones (Shone, 1987). Por lo tanto, es más fácil separar la salmuera del sólido.

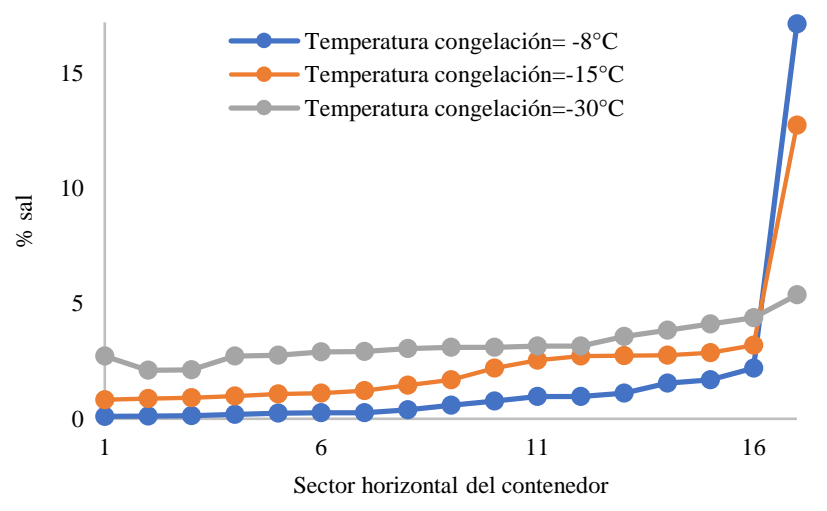

Figura $7 \quad \%$ Salinidad en función del sector de contenedores para diversas velocidades de congelación, con $\mathrm{L}=15$ pulgadas

Para estudiar el movimiento salino de todas las geometrías sin aislamiento, calculamos la concentración de sal en diferentes sectores. Los resultados se muestran en la Tabla 1._Para sectores concéntricos (tipo A), la dirección de difusión de iones salinos es desde el centro hacia la superficie del hielo formado (Tabla 1). El resultado concuerdan con (Rahman et al., 2007). Se debe a la baja solubilidad de la sal en agua sólida. Cuando el hielo comienza a formarse, la sal se rechaza a los niveles exteriores lentamente, hasta que la congelación total del agua.

\begin{tabular}{|l|r|}
\hline \multicolumn{1}{|c|}{ Sector } & gr NaCl/L \\
\hline 1 (superficie de hielo formado) & 49.28 \\
\hline 2 & 47.33 \\
\hline 3 & 30.48 \\
\hline 4 & 15.34 \\
\hline 5 & 4.263 \\
\hline 6 (centro del hielo formado) & 1.327 \\
\hline
\end{tabular}

Tabla 1 Salinidad de la superficie hacia el interior del hielo formado

Además, para los sectores horizontales (tipo B), se encuentra que, la dirección de difusión de iones salinos es de la parte inferior a la parte superior del contenedor (Tabla 2). La difusión se ve afectada por el fenómeno de flotabilidad y el contacto del recipiente con la superficie del congelador más frío. Este contacto permite un gradiente térmico que mejora el movimiento hacia la superficie del contenedor. Los resultados del corte tipo A se ven afectados por este movimiento, razón por la cual el nivel concéntrico más alto (1) contiene la sal concentrada. Para corroborar este resultado, se probó el corte tipo $\mathrm{C}$, y como se puede ver en la Tabla 2, la difusión de sal comienza desde los sectores inferiores hasta la parte superior del contenedor.

\begin{tabular}{|c|r|}
\hline Sector & (gr $\mathbf{~ N a C l} / \mathbf{L})$ \\
\hline $\mathrm{A} 1$ & 13 \\
\hline $\mathrm{A} 2$ & 12.9 \\
\hline $\mathrm{A} 3$ & 13 \\
\hline $\mathrm{A} 4$ & 12.2 \\
\hline $\mathrm{B} 1$ & 3.5 \\
\hline $\mathrm{B} 2$ & 2.5 \\
\hline $\mathrm{B} 3$ & 2.7 \\
\hline $\mathrm{B} 4$ & 2.9 \\
\hline $\mathrm{C} 1$ & 1.7 \\
\hline $\mathrm{C} 2$ & 1.2 \\
\hline $\mathrm{C} 3$ & 1.4 \\
\hline $\mathrm{C} 4$ & 2 \\
\hline
\end{tabular}

Tabla 2 Salinidad observada Corte tipo C, desplazamiento de abajo a arriba

\section{Alcances y recomendaciones}

Los resultados de este estudio experimental ayudarán a diseñar y construir prototipos de desalación por congelación más adecuados, de alto rendimiento para plantas industriales. Consecuentemente, se puede contribuir al suministro de agua dulce a las regiones costeras de los países desfavorecidos, con bajos requerimientos energéticos y con viabilidad económica. El estudio experimental debe complementarse con un estudio teórico para validar y refinar los resultados. 
Una simulación del modelo teórico permitiría controlar en tiempo real las tasas de refrigeración adecuadas de acuerdo con las características de la salmuera cada vez más concentrada de tal manera que se pueda optimizar la desalinización de congelación.

\section{Conclusiones}

En resumen, analizamos experimentalmente la difusión salina durante la desalación del agua por congelación. Utilizamos aislamiento térmico para tener una difusión térmica y de masa unidimensional. La sal se desplaza de la sección más fría a la más cálida y se acumula hasta que llega a un punto en el que la salmuera no se logra congelar. Esto facilita separación del agua congelada pura de la salmuera líquida concentrada.

Además, estudiamos el impacto de la longitud del contenedor en la difusión de la sal. Se encontró que, la difusión de la sal aumenta con el aumento de la longitud del recipiente. Además, los experimentos se llevaron a cabo a diferentes temperaturas. La menor velocidad de congelación mejora la difusión salina, ya que da oportunidad a la masa de moverse antes de quedar atrapado dentro del hielo. De esta manera, es posible eliminar el 78,8\% de la sal en el hielo formado a $-8^{\circ} \mathrm{C}$. Además, se estudió el impacto de la geometría en la difusión salina. Se encuentra que, para todas las geometrías, la dirección de difusión de iones salinos es de la parte inferior a la parte superior del hielo formado.

El análisis experimental realizado nos ayuda a comprender de una mejor manera el desplazamiento salino durante la congelación del agua de mar, que es uno de los principales problemas que presenta la tecnología. Por lo tanto, nos da información valiosa para optimizar el proceso de desalinización mediante la congelación.

\section{Referencias}

Ahmad, M., \& Williams, P. (2011). Assessment of desalination technologies for high saline brine applications - Discussion Paper. Desalination and Water Treatment, 30(1-3), 22-36. https://doi.org/10.5004/dwt.2011.1374
CastilloTéllez, B. (2017). Análisis teórico y experimental de los fenómenos de transporte asociados a la difusión salina durante la congelación de agua de mar para su potabilización, utilizando energía solar (Universidad Nacional Autónoma de México). Retrieved from http://132.248.9.195/ptd2017/mayo/0758838/In dex.html

CastilloTéllez, B., Castillo Téllez, M., González Chan, J. de J., \& Sarracino Martínez, O. (2018). Experimental of Seawater Desalination using Thermosolar Energy. European Journal of Sustainable Development, 7(4), 74-80. https://doi.org/10.14207/ejsd.2018.v7n3p74

Chabarov, A., \& Aider, M. (2014). Mathematical modeling and experimental validation of the mass transfer during unidirectional progressive cryoconcentration of skim milk. Innovative Food Science and Emerging Technologies, 21, 151-159. https://doi.org/10.1016/j.ifset.2013.08.001

Cole, D. M., \& Shapiro, L. H. (1998). Observations of brine drainage networks and microstructure of first-year sea ice. Journal of Geophysical Research, 103(C10), 21739. https://doi.org/10.1029/98JC01264

Fujioka, R., Wang, L. P., Dodbiba, G., \& Fujita, T. (2013). Application of progressive freezeconcentration for desalination. Desalination, 319 , 33-37. https://doi.org/10.1016/j.desal.2013.04.005

Gao, L., Yoshikawa, S., Iseri, Y., Fujimori, S., \& Kanae, S. (2017). An Economic Assessment of the Global Potential for Seawater Desalination to 2050. Water, 9(10), 763. https://doi.org/10.3390/w9100763

Gao, W., \& Shao, Y. (2009). Freeze concentration for removal of pharmaceutically active compounds in water. Desalination, 249(1), 398-402. https://doi.org/10.1016/j.desal.2008.12.065

Ghaffour, N., Missimer, T. M., \& Amy, G. L. (2013). Technical review and evaluation of the economics of water desalination: Current and future challenges for better water supply sustainability. Desalination, 309, 197-207. https://doi.org/10.1016/j.desal.2012.10.015 
Grange, B. W., Viskanta, R., \& Stevenson, W. H. (1976). Diffusion of heat and solutescraped surface freezer during freezing of salt solutions. International Journal of Heat and Mass Transfer, 19, 373-384. https://doi.org/10.1016/0017-9310(76)90093-4

Halde, R. (1980). Concentration of impurities by progressive freezing. Water Research, 14(6), 575-580. https://doi.org/10.1016/00431354(80)90115-3

Johnson, W. E. (1979). Indirect freezing. Desalination, 31(1-3), 417-425. https://doi.org/10.1016/S0011-9164(00)885458

Jungwirth, P., Vrbka, L., \& Jungwirth, P. (2005). Brine rejection from freezing salt solutions: A molecular dynamics study. Physical Review Letters, 95(September), 1-4. https://doi.org/10.1103/PhysRevLett.95.148501

Karagiannis, I. C., \& Soldatos, P. G. (2008). Water desalination cost literature: review and assessment. Desalination, 223(1-3), 448-456. https://doi.org/10.1016/j.desal.2007.02.071

Li, C., Goswami, Y., \& Stefanakos, E. (2013). Solar assisted sea water desalination: A review. Renewable and Sustainable Energy Reviews, 19, 136-163.

https://doi.org/10.1016/j.rser.2012.04.059

M.S. Rahman, M. Ahmed, X. D. C. (2007). Freezing-Melting Process in Liquid Food Concentration. In Handbook of Food Preservation (10th ed., pp. 667-690). New York, NY 10016: CRC Press.

Melinder, A., \& Ignatowicz, M. (2015). Properties of seawater with ice slurry use in focus. International Journal of Refrigeration, 52 , $51-58$ https://doi.org/10.1016/j.ijrefrig.2014.12.022

Melinder, Ake. (2007). Thermophysical Properties of Aqueous Solutions Used as Secondary Working Fluids. Royal Institute of Technology, KTH, Stockholm, Sweden.

Peñate, B., \& García-Rodríguez, L. (2012). Current trends and future prospects in the design of seawater reverse osmosis desalination technology. Desalination, 284, 1-8. https://doi.org/10.1016/j.desal.2011.09.010

ISSN -2410-3950

ECORFAN® Todos los derechos reservados
Rahman, M. S., Ahmed, M., \& Chen, X. D. (2007). Freezing melting process and desalination: review of present status and future prospects. International Journal of Nuclear Desalination, 2(3), 253. https://doi.org/10.1504/IJND.2007.013549

Reddy, K. V., \& Ghaffour, N. (2007). Overview of the cost of desalinated water and costing methodologies. Desalination, 205(1-3), 340353. https://doi.org/10.1016/j.desal.2006.03.558

Rogers, G. F. C., \& Mayhew, Y. R. (1995). Thermodynamic and Transport Properties of Fluids. 1-28.

Shirai, Y., Wakisaka, M., Miyawaki, O., \& Sakashita, S. (1998). Effect of seed ice on formation of tube ice with high purity for a freeze wastewater treatment system with a bubble-flow circulator. Water Research, 33, 1325-1329. https://doi.org/10.1016/S00431354(98)00335-2

Shone, R. D. C. (1987). The freeze desalination of mine waters *. J. S. At,. Inst. Min. Metal/, 87(4), 107-112.

Subramani, A., \& Jacangelo, J. G. (2015). Emerging desalination technologies for water treatment: A critical review. Water Research, 75 , 164-187.

https://doi.org/10.1016/j.watres.2015.02.032

Wiegandt, H., \& Berg, R. Von. (1980). Myths about freeze desalting. Desalination, 33(July 1979), 287-297. Retrieved from http://www.sciencedirect.com/science/article/pi i/S0011916400885719

Williams, P. M., Ahmad, M., Connolly, B. S., \& Oatley-Radcliffe, D. L. (2015). Technology for freeze concentration in the desalination industry. Desalination, 356, 314-327. https://doi.org/10.1016/j.desal.2014.10.023

Yu, T., Ma, J., \& Zhang, L.-Q. (2007). Factors affecting ice crystal purity during freeze concentration process for urine treatment. Journal of Harbin Institute of Technology (New Series), 14(5). 